\title{
Preface
}

\section{The Quest for Diagnostic Excellence in Critical Care}

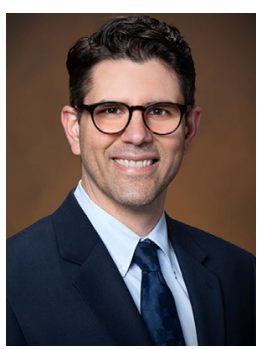

Paul A. Bergl, MD

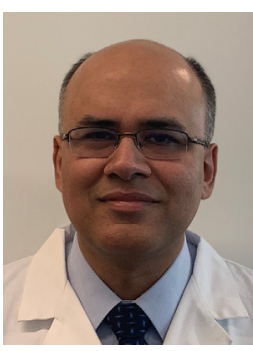

Rahul S. Nanchal, MD

Editors

The modern intensive care unit (ICU) abounds with complexity. We support multiple failing organ systems, often among patients with a bewildering array of comorbidities and complications. We deal with the challenging tripartite intersection of what is possible, what patients and families desire, and what is reasonable. To stabilize deranged physiology, we apply an armamentarium of sophisticated technologies that in turn generate patient data ad infinitum: vital signs, laboratory test results, diagnostic images, ventilator parameters, measures of cardiac output, fluid balances, and much more.

This immense body of patient data notwithstanding, we as intensivists face significant challenges in arriving at accurate diagnoses for our critically ill patients. Our patients often cannot provide a cogent history of their illness, and we must rely on imperfect information provided second or third hand. Furthermore, by the time a patient arrives in the ICU, they usually have been subjected to a number of interventions that affect their physiology, change the natural history of their illness, and muddy our own judgment. We practice at a brisk pace, and the cognitively taxing nature of our work is compounded by the high stakes of stabilizing patients on the brink of death and the charged emotions that emanate from our patients, families, staff, and colleagues.

Unsurprisingly, the labyrinthian and stressful ICU environment is rife with errors in judgment and diagnosis. Reasonable estimates suggest that at least $5 \%$ of deaths in the ICU result from fatal misdiagnoses. Nonfatal diagnostic errors, which occur in an additional $5 \%$ to $10 \%$ of ICU patients, also confer significant morbidity, generate immense financial costs, and have heavy emotional tolls.

This issue of the Critical Care Clinics explores diagnostic excellence in the ICU from various perspectives. Because discussing the topic requires a shared dialect and foundational knowledge, we first review definitions of diagnostic errors, methods for 
identifying them, and the epidemiology of diagnostic error in critical care. We then delve into the science of human cognition and provide a contemporary understanding of how intensivists arrive at a diagnosis and the pitfalls that lead to reasoning errors.

In the latter half of this issue, we propose potential solutions to mitigate errors and to promote diagnostic excellence in the ICU. We introduce the concept of diagnostic stewardship and examine how systems can be designed to improve the appropriateness, and accordingly, the utility and accuracy, of diagnostic testing. We then return to human cognition and discuss practical strategies and educational interventions to improve diagnostic reasoning. Building upon strategies targeted at improving individual clinician's diagnostic performance, we consider how to optimize teamwork and ultimately how to reengineer our health care delivery systems to improve diagnosis. We discuss how structured reflection, feedback, and routine diagnostic auditing could operate at both the individual and the systems level of care to reduce cognitive failures. Finally, we conclude with a research agenda that hones in on aspects of our care delivery systems that affect the diagnostic process.

Diagnostic errors remain unacceptably common in the ICU. We hope that this issue of Critical Care Clinics will galvanize the critical care community to develop pragmatic solutions to this neglected area of patient safety. Our patients deserve the safest, most-effective care possible; diagnostic excellence is the backbone of such care.

Paul A. Bergl, MD

Gundersen Lutheran Medical Center 1900 South Avenue, Mail Stop LM3-001

La Crosse, WI 54601, USA

Rahul S. Nanchal, MD

Medical College of Wisconsin

Division of Pulmonary, Critical Care, and Sleep Medicine 8th Floor, Hub for Collaborative Medicine

8701 West Watertown Plank Road Milwaukee, WI 53226, USA

E-mail addresses:

pabergl@gundersenhealth.org (P.A. Bergl) rnanchal@mcw.edu (R.S. Nanchal) 